RUNNING HEAD: P-CURVING PROFESSORS AND ELDERLY

\title{
Professors Are Not Elderly: Evaluating the Evidential Value of Two Social Priming Effects through P-Curve Analyses
}

\author{
Daniël Lakens \\ Eindhoven University of Technology
}

Word count: 2887

Keywords: P-curve, Social Priming, Statistical Power, Meta-Analysis

Author Note: I would like to thank Sander Koole for feedback on an earlier version of this manuscript.

Correspondence can be addressed to Daniël Lakens, Human Technology Interaction Group, IPO 1.33, PO Box 513, 5600MB Eindhoven, The Netherlands. E-mail: D.Lakens@tue.nl. The analyses and $p$-curve disclosure tables are available at http://osf.io/3urp2/ 
$P$-Curving Professors and Elderly 2

\begin{abstract}
It is possible that the number of false positives in the literature is much greater than is desirable due to a combination of low statistical power, publication bias, and flexibility when analyzing data. Recently, some researchers have argued the replicability crisis in social priming research is greatly exaggerated (Dijksterhuis, 2014; Stroebe \& Strack, 2014). To quantify the extent to which flexibility during the data analysis is a real problem, I present two $p$-curve analyses that examine the evidential value of research lines on professor priming and elderly priming. The results indicate studies examining elderly priming are $p$-hacked, while studies examining professor priming contain evidential value. I believe a polarized discussion about whether social priming is true or not, whether direct replications or conceptual replications are preferable, or whether methodological rigor or theory development is needed is unlikely to lead to scientific progress. Instead, we have to metaanalytically evaluate individual effects based on their evidential value, and collaboratively examine what is likely to be true.
\end{abstract}


Significant results in the published literature can reflect either true effects or false positives. It is possible that the number of false positives in the literature is much greater than is desirable due to a combination of low statistical power, publication bias, and flexibility during the data analysis (e.g., Ioannides, 2005; Simmons, Nelson, \& Simonsohn, 2011). Recently, some researchers have argued that "there are good reasons to believe that the fear that psychology is infested with false positives is largely unnecessary” (Dijksterhuis, 2014, p. 73), "the claim of a replicability crisis is greatly exaggerated” (Stroebe \& Strack, 2014, p. 59), and that “we are at risk of becoming methodological fetishists” (Ellemers, 2013, p. 3). Are these researchers correct? Is there little reason to worry?

One way to objectively answer this question is to quantify the evidential value of a line of research that examines a specific hypothesis. In addition to large scale replication efforts (e.g., Open Science Collaboration, 2012), we can estimate these probabilities by performing meta-analyses on performed research. Traditional meta-analyses are one approach, but suffer from publication bias. $P$-curve analysis is a recently developed metaanalytic procedure (Simonsohn, Nelson, Simmons, 2014) that is unaffected by publication bias. $P$-curve analysis can differentiate between published findings that should increase our prior belief that a specific hypothesis is true, and findings that should not increase our prior belief that a specific hypothesis is true.

The two $p$-curve analyses presented here showcase the benefits of $p$-curve analyses to improve our understanding of what is likely to be true. In light of recent close replication studies that did not provide support the hypothesis (Doyen, Klein, Pichon, \& Cleeremans, 2012; Shanks et al, 2013) Stroebe and Strack (2014) recently discussed the importance of conceptual replications (and questioned the usefulness of close replications) when examining the effects of elderly primes and professor primes on human behavior. Studies examining elderly priming predict that activating the stereotype of elderly will make people behave 
more slowly (e.g., Bargh, Chen, \& Burrows, 1996). Studies examining professor priming predict that activating the stereotype of a professor will make people work harder and perform better on a general knowledge test (e.g., Dijksterhuis \& Van Knippenberg, 1998). I believe that both close as conceptual replications have their place in the empirical cycle (see Koole \& Lakens, 2012; Nosek \& Lakens, 2014), but that it is especially important to evaluate the outcome of replication studies in their meta-analytic context.

Given that direct replications are scarce in psychology (Fanelli, 2012), lines of research primarily consist of conceptual replications (LeBel \& Peters, 2011). In situations where there is strong evidential value for a specific hypothesis based on a series of conceptual replications, failed close replications should instigate a discussion about potential moderators. On the other hand, if a line of conceptual replications lacks evidential value, a lack of an effect in a close replication of one of these experiments is much more likely to occur, and such an outcome should increase our doubt about the likelihood the hypothesis is true. Which interpretation is more appropriate can only be determined by weighing the informational value of the research line. $P$-curve analyses are one way to achieve this.

\section{P-Curve as a Key to the File Drawer}

The distribution of $p$-values between .00 and .05 retrieved from published findings should be right-skewed when the alternative hypothesis is true (i.e., more $p$-values between .00 and .01 than between .04 and .05). $P$-values are uniformly distributed when the nullhypothesis is true. If you would simulate hundreds of studies (for example using ESCI, Cumming, 2012) where two populations are compared with identical mean scores on the variable of interest, but where due to variance the sample means always show some random variation, every $p$-value between .00 and .99 is equally likely to be observed. As a consequence, in $5 \%$ of the studies the $p$-value will be lower than $p=.05$ (a Type 1 error). 
By examining the $p$-value distribution of a set of studies it can be determined whether the findings contain evidential value (i.e., a right-skewed distribution, in line with the expected $p$-value distribution of a true effect) or lack evidential value (i.e., a uniform distribution, in line with the expected $p$-value distribution of no effect). A third possibility is that the distribution of $p$-value is left-skewed (i.e., there are relatively many $p$-values between .04 and .05 , but relatively few between .00 and .01 ), which is an indication that the results are not observed by chance, but that researchers actively selected one of the possible tests they could perform on the data (e.g., by excluding participants, selectively reporting variables, etc.) because this test yielded a significant result. If an effect exists, $p$-values between .04 and .05 are relatively unlikely, and it is improbable many of these $p$-values are observed in a line of research. Indeed, in high powered experiments, $p$-values between .04 and .05 are relatively more likely when the null-hypothesis is true than when the null-hypothesis is false (Lakens \& Evers, 2014; Sellke, Bayarri, \& Berger, 2001). Because a significantly left-skewed ( $p$ hacked) distribution and an uniform $p$-curve distribution both indicate a lack of evidential value, of primary interest when examining lines of studies meta-analytically is whether the $p$ curve is right-skewed (i.e., contains evidential value) or not.

\section{P-Curves of the Effects of Elderly Primes and Professor Primes}

Stroebe and Strack (2014) suggest that failed replications by Doyen, Klein, Pichon, and Cleeremans (2012) and Shanks et al (2013) of the effects of elderly and professor primes were 'astounding' in light of earlier conceptual replications. In their appendix they briefly review conceptual replications of elderly prime and professor prime effects. Similarly, Dijksterhuis (2014) suggests that 'hundreds of papers cannot be erased by the mere flick of a skeptic magic wand'. Both these arguments are the meta-analytical equivalent of votecounting procedures, where the presence of an effect is inferred from the number of successful observations in the literature. Due to publication bias vote-counting procedures are 
fundamentally flawed and should not be used (Borenstein, Hedges, Higgins, \& Rothstein, 2006). Instead of summarizing findings in support of the presence of an effect in a narrative review, theoretical reviews that want to draw conclusions about the likelihood of a theoretical idea should rely on meta-analytical techniques. Whereas traditional meta-analyses require researchers to search for unpublished manuscripts, all the required information to perform a $p$-curve analysis is readily available in the reviewed literature, which makes it a more practical procedure to complement theoretical review articles. I have $p$-curved the studies cited by Stroebe and Strack (2014) ${ }^{1}$ to demonstrate how a meta-analytic evaluation of the evidential value of research lines using $p$-curve analyses can be used to interpret the most likely reason replication studies failed to observe an hypothesized effect. These analyses illustrate that lines of research consisting of conceptual replications do not necessarily provide support for a theoretical idea - they do so only if they have evidential value.

The data for the $p$-curve analyses presented below is reported in a $p$-curve disclosure table, which can be found at the Open Science Framework, http://osf.io/3urp2/. All $p$-values included in the analyses are statistically independent. Whenever multiple comparisons between conditions were possible (e.g., 3-cell designs consisting of a strong manipulation vs. weak manipulation vs. control condition) the test which yielded the lowest $p$-value was conservatively included in the reported analysis (and additional tests were coded in the disclosure table under 'robustness result'). Following the recommendations by Simonsohn et al., (2014) whenever a cross-over interaction was predicted both $p$-values for simple effects were included in the analysis, and the $p$-value for the interaction was used when attenuated interactions were predicted.

\section{Elderly Priming}

This $p$-curve analysis includes the empirical studies reviewed by Stroebe and Strack (2014) that examined effects of elderly primes on human behavior (Aarts \& Dijksterhuis, 
2002; Bargh et al., 1996; Cesario, Plaks, \& Higgins, 2006; Dijksterhuis, Spears, \& Lepinasse, 2001; Kawakami, Young, \& Dovidio, 2002; Ku, Wang, \& Galinsky, 2010; Macrae et al., 1998; Mussweiler, 2006). For one of the studies, test statistics were not reported in sufficient detail to be included in the $p$-curve analysis, and the final analysis consisted of $18 p$-values from 17 studies reported in 8 articles. The results of the $p$-curve analysis indicate the distribution is significantly left-skewed, $X^{2}(36)=51.82, p=.043$ (see the black line in Figure 1), which indicates at least some of the studies were $p$-hacked (see Simonsohn et al., 2014). However, as discussed earlier, $p$-values between .04 and .05 at best provide very weak support for a hypothesis, and the significance of the test for left-skew hinges on a single $p$ value. The most important conclusion is that the line of research clearly lacks evidential value, $X^{2}(36)=72.42, p=.0003$, irrespective of whether the studies were significantly $p$ hacked or not.

Based on the distribution of $p$-values, the published studies do not provide support for the hypothesis that elderly primes influence behavior. Furthermore, there is at least some indication that the results reported in the literature are not just Type 1 errors due to random variation in the data, but that the reported tests suffer from researchers' degrees of freedom (Simmons et al., 2011) and were actively selected from larger number of performed tests because the reported tests yielded significant results. Although a significantly $p$-hacked line of research is a reason to worry, it is possible at least some researchers who contributed to this line of research reported the results from pre-planned analyses. Therefore, conclusions about the use of researchers' degrees of freedom at the group level cannot be generalized to the individual level.

The average effect size across the 18 effects reveals an $r$ of .29 (for calculations, see Lakens, 2013). Remember that due to publication bias the true effect size might very well be much lower (and perhaps even 0). However, even based on the likely overestimated effect 
size of $r=.29$ (or Cohen's $d=0.61$ ), the minimum sample size to achieve a statistical power of .80 would require 44 participants in each between subject condition. The average sample size in each between subject condition in the reviewed studies was 22, and none of the performed experiments had sufficient power to detect an effect of $r=.29$.

Professor Priming

This $p$-curve analysis includes the empirical studies reviewed by Stroebe and Strack (2014) that examined effects of professor primes on human behavior (Bry, Follenfant, \& Meyer, 2008; Dijksterhuis \& Van Knippenberg, 1998; Galinsky, Wang, \& Ku, 2008; Haddock, Macrae, \& Fleck, 2002; Hansen \& Wänke, 2009; LeBoeuf \& Estes, 2004; Lowery, Eisenberger, Hardin, \& Sinclair, 2007; Nussinson, Seibt, Häfner, \& Strack, 2010). Because two of the $p$-values were not significant at the $p=.05$ level, the final selection consisted of 18 $p$-values from 16 studies reported in 8 articles. The results of the $p$-curve analysis (see Figure 2) indicate the studies contain evidential value, $X^{2}(40)=73.92, p<.001$ (see Simonsohn et al., 2014). The observed distribution of $p$-values mirrors the expected distribution of $p$-values for a true effect.

The average effect size across all 22 effects (including not significant results) reveals an $r$ of .36 (which is almost certainly an overestimation due to publication bias). Based on the most likely overestimated effect size of $r=.36$ (or Cohen's $d=0.77$ ), the minimum sample size to achieve a statistical power of .80 would require 28 participants in each between subject condition. The average sample size in each between subject condition in the reviewed studies was 24, and six of the performed experiments had sufficient power to detect an effect of $r=.36$.

\section{General Discussion}

When evaluating the evidential value of studies that examined effects of elderly primes and professor primes, two divergent $p$-distributions were evident. Where studies that 
examined effects of professor primes contain evidential value, studies that examined effects of elderly primes were significantly p-hacked (Simmons et al., 2011; Simonsohn et al., 2014). Both effects can still turn out to be true or false, but at this moment, there are quantifiable and objective reasons to differentiate between the likelihood these specific hypotheses are true.

These findings speak against the idea that false positives are nothing to worry about (Dijksterhuis, 2014). Furthermore, although conceptual replications are in principle an excellent way to assess the robustness of theoretical ideas, in practice conceptual replications can inflate the Type 1 error rate whenever only significant results are published, and nonsignificant results are seen as failed pilot studies that end up in the file drawer (LeBel \& Peters, 2011). Conceptual replications only support and extend a hypothesis if they contain evidential value. Narrative reviews that present conceptual replications as support for an hypothesis should meta-analytically evaluate the evidential value of these studies. Since $p$ curve analyses can be performed solely based on information contained in the published literature, they are an efficient and feasible meta-analytic technique to use when reviewing the literature. Finally, the results speak against generalized claims about the replicability of social priming (e.g., Pashler, Coburn, \& Harris, 2012). Instead, it seems to be more productive to meta-analytically evaluate individual hypotheses on their informational value (see Lakens \& Evers, 2014).

Through false polarization (Robinson, Keltner, Ward, \& Ross, 1995) a group of 'skeptics' and a group of 'believers' has emerged in the discussion about the reliability of social priming effects. The current $p$-curve analyses illustrate why polarized discussions about broad theoretical hypotheses such as whether social priming exists will not lead to scientific progress. Some priming effects, such as the finding that professor primes influence performance on general knowledge tests, seem to have something going for them and deserve our empirical attention. The failed replications by Shanks et al. (2013) are somewhat 
unexpected, although it should be noted that 8 out of 9 replication studies performed by Shanks and colleagues were underpowered, even when we calculate power based on the effect size from the published literature (i.e., had less than 28 participants in each condition). Underpowered studies have low informational value and yield widely varying effect size estimates (see Lakens \& Evers, 2014), and are especially problematic in replication studies (e.g., Zwaan, 2014).

Other hypotheses about priming, such as the predicted effect of elderly primes on behavior, have not become more likely after collecting empirical data. There could be several reasons for this lack of empirical support: The used methodology might be ill-suited to demonstrate the phenomenon reliably, the effect might by considerably smaller than the performed studies were powered to detect, or the effect might not exist. Given the lack of evidential value for the hypothesis that elderly primes influence the speed of behavior, the replication by Doyen et al (2012) that did not reveal an effect of elderly primes should not be a surprise, and seems most parsimoniously explained by the idea that the original finding was a Type 1 error. The $p$-curve indicates researchers' degrees of freedom are problematic in some lines of research. It is important to identify such research lines if we want to develop better theories of the mechanisms underlying social priming effects (e.g., Cesario, 2014).

A more interesting question than whether evidential value is present or absent, is why these two ostensibly similar priming effects differ in their evidential value. Although any explanation of why professor priming seems to have something going for it but elderly priming does not will require further research, let’s start by examining the differences between elderly priming studies and professor priming studies in more detail. One difference in the priming manipulation is that professor priming often occurred by asking people to list behaviors and characteristics of professors (or groups stereotypically associated with less intelligence), while prime activation in elderly priming studies was typically more subtle, and 
accomplished by (briefly) showing words, or asking participants to categorize stimuli such as pictures as elderly or not. Therefore, construct activation might have been in general stronger in the professor priming studies than in the elderly priming studies. A second difference might be that professors are more strongly associated with intelligence than elderly are associated with slowness. Finally, activated constructs might have more influence on motivation (e.g., the willingness to think long and hard about general knowledge questions) than on the speed with which behavior is performed (e.g., walking down a corridor).

All these possibilities seem to be in line with theoretical models of the effects of primes on behavior and motivation (e.g., Loersch \& Payne, 2011), and researchers interested in theory development might be inspired by these or additional differences between elderly priming and professor priming to examine the boundary conditions or mechanisms underlying behavioral priming. Such future research might even be able to determine the conditions under which elderly primes do reliably influence behavior. After all, as McGuire (1983, p. 7) has remarked, all theories are right, and the goal of empirical discovery is 'clarifying circumstances under which the hypothesis is true and those under which it is false'. To achieve this, we need to perform both close as conceptual replications, and publish the results irrespective of the significance level of the observed effect.

The current demonstration of how a novel statistical technique can inspire new theoretical questions nicely illustrates how methodological rigor and theory development, another false polarization in recent discussions about reproducibility (e.g., Ellemers, 2013), actually go hand in hand. Instead of polarized discussions, let's get back to work, collectively and collaboratively, and examine what is likely to be true. 


\section{Figures}

Figure 1. P-curve distribution of elderly priming studies (black solid line), compared to the expected distribution when the null hypothesis is true (red dotted line) or the alternative hypothesis is true and studies were powered at 33\% (green striped line).

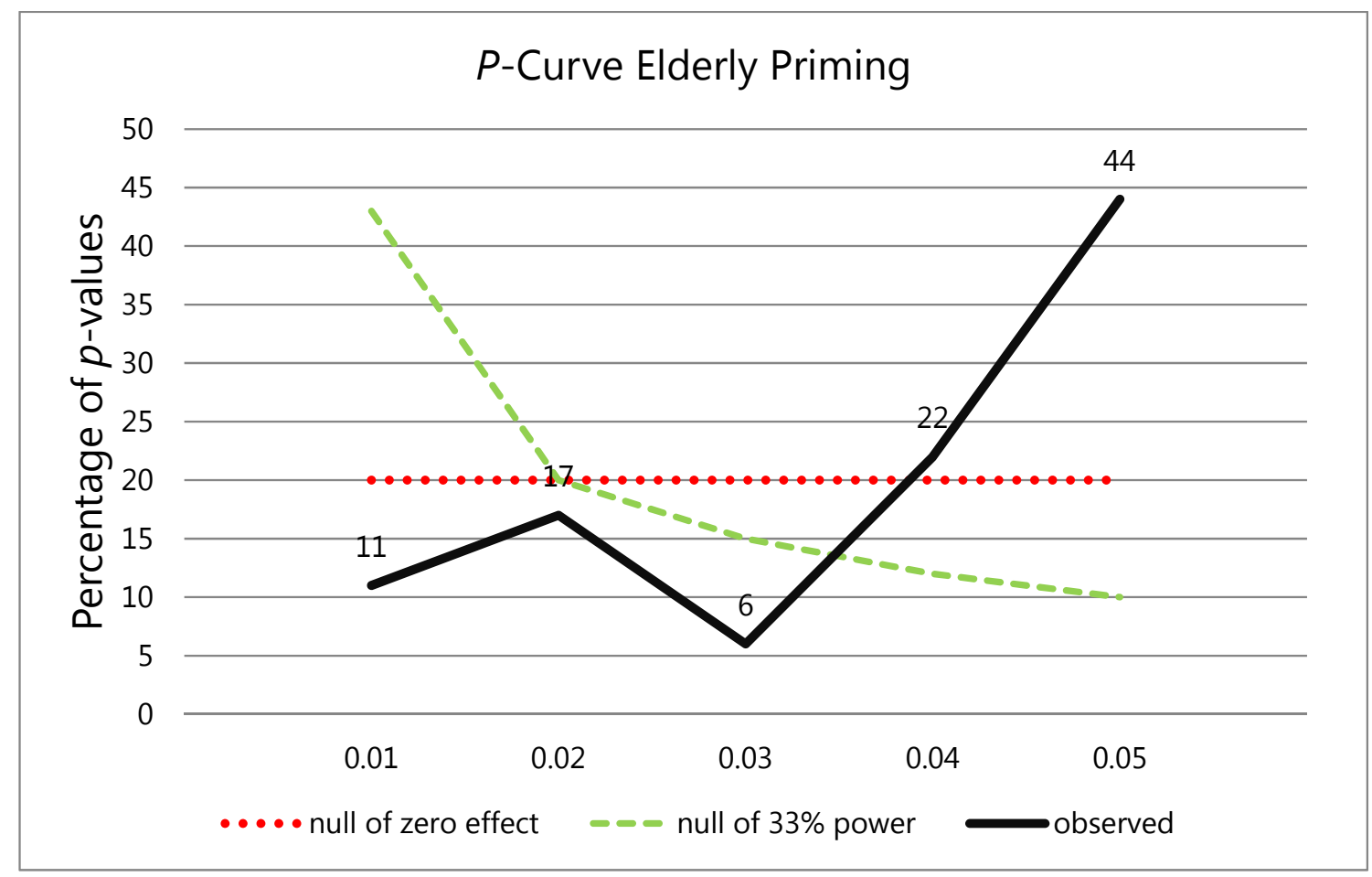


Figure 2. P-curve results (black solid line) of professor priming studies, compared to the expected distribution when the null hypothesis is true (red dotted line) or the alternative hypothesis is true and studies were powered at 33\% (green striped line)

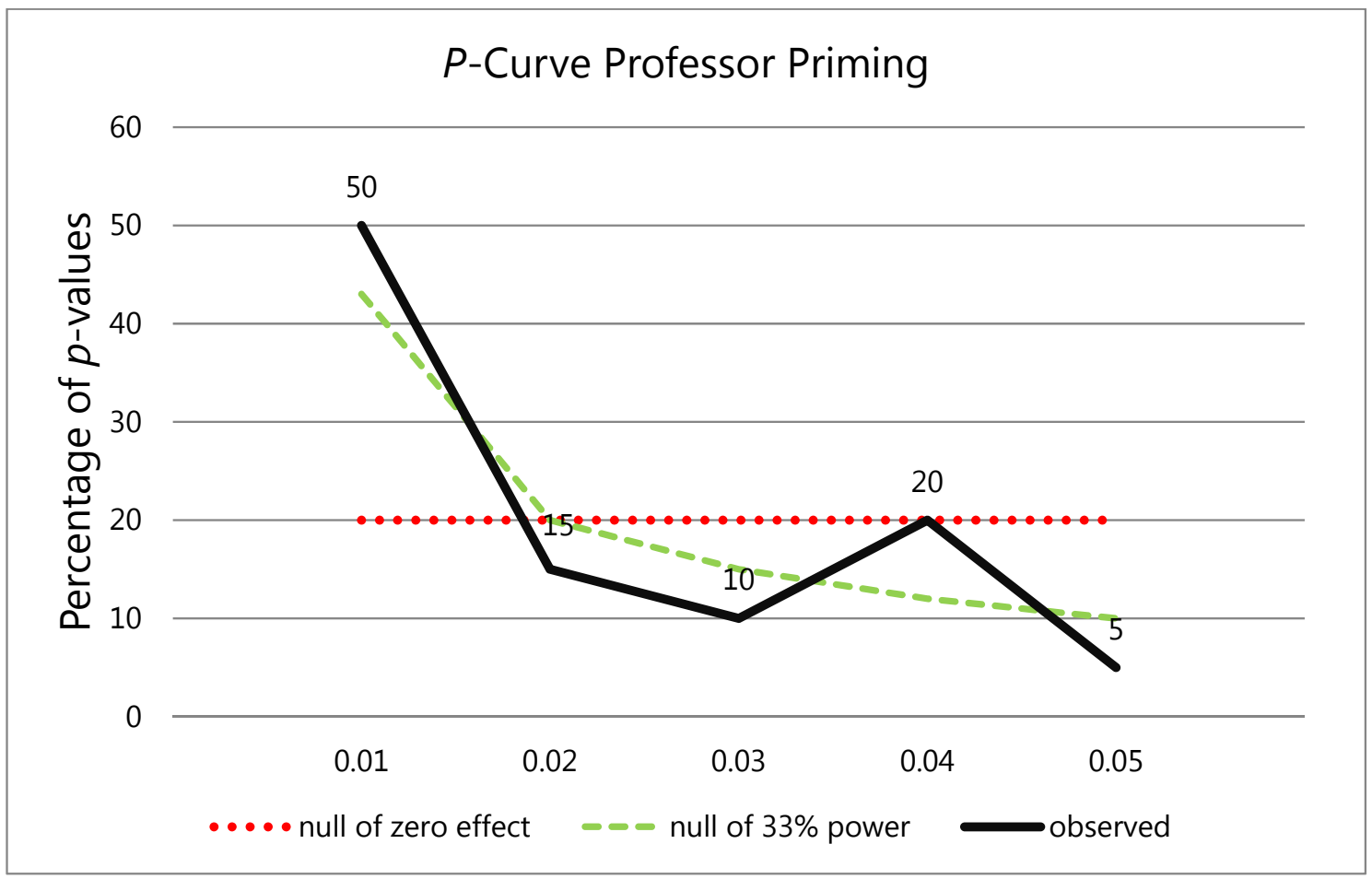


P-Curving Professors and Elderly 14

\section{References}

Aarts, H., \& Dijksterhuis, A. (2002). Category activation effects in judgment and behaviour: The moderating role of perceived comparability. British Journal of Social Psychology, 41, 123-128.

Bargh, J. A., Chen, M., \& Burrows, L. (1996). Automaticity of social behavior: Direct effects of trait construct and stereotype-activation on action. Journal of Personality and Social Psychology, 71, 230-244.

Borenstein, M., Hedges, L. V., Higgins, J. P., \& Rothstein, H. R. (2011). Introduction to meta-analysis. Hoboken, NJ: Wiley.

Bry, C., Follenfant, A., \& Meyer, T. (2008). Blonde like me: When self-construals moderate stereotype priming effects on intellectual performance. Journal of Experimental Social Psychology, 44, 751-757.

Cesario, J. (2014). Priming, replication, and the hardest science. Perspectives on Psychological Science, 9, 40-48.

Cesario, J., Plaks, J. E., \& Higgins, T. E. (2006). Automatic social behavior as motivated preparation to interact. Journal of Personality and Social Psychology, 90, 893-910.

Cumming, G. (2012). Understanding the new statistics: Effect sizes, confidence intervals, and meta-analysis. New York: Routledge.

Dijksterhuis, A. (2014). Welcome back theory! Perspectives on Psychological Science, 9, 7275.

Dijksterhuis, A., Spears, R., \& Lepinasse, V. (2001). Reflecting and deflecting stereotypes: Assimilation and contrast in impression formation and automatic behavior. Journal of Experimental Social Psychology, 37, 286-299. doi:10.1006/ jesp.2000.1449 
Dijksterhuis, A., \& van Knippenberg, A. (1998). The relation between perception and behavior or how to win a game of trivial pursuit. Journal of Personality and Social Psychology, 74, 865-877.

Doyen, S., Klein, O., Pichon, C.-L., \& Cleeremans, A. (2012). Behavioral priming: It’s all in the mind, but whose mind? PLoS ONE, 7, e29081. doi:10.137/journal.pone.0029081

Ellemers, N. (2013). Connecting the dots: Mobilizing theory to reveal the big picture in social psychology (and why we should do this). European Journal of Social Psychology, 43, 1-8. DOI: 10.1002/ejsp.1932

Fanelli, D. (2012). Negative results are disappearing from most disciplines and countries. Scientometrics, 90, 891-904.

Galinsky, A. D., Wang, C. S., \& Ku, G. (2008). Perspectivetakers behave more stereotypically. Journal of Personality and Social Psychology, 95, 404-419.

Haddock, G., Macrae, C. N. \& Fleck, S. (2002). Syrian science and smart supermodels: On the when and how of perception-behavior effects. Social Cognition, 20, 461-481.

Hansen, J., \& Wänke, M. (2009). Think of capable others and you can make it! Self-efficacy mediates the effect of stereotype activation on behavior. Social Cognition, 27, 76-88.

Ioannidis, J. P. (2005). Why most published research findings are false. PLoS Medicine, 2, e124. doi:10.1371/journal.pmed.0020124.

Kawakami, K. L., Young, H., \& Dovidio, J. F. (2002). Automatic stereotyping: Category, trait, and behavioral activations. Personality and Social Psychology Bulletin, 28, 315.

Koole, S. L., \& Lakens, D. (2012). Rewarding replications: A sure simple way to improve psychological science. Perspectives on Psychological Science, 7, 608-614. 
Ku, G., Wang, C. S., \& Galinsky, A. D. (2010). Perception through a perspective-taking lens: Differential effects on judgments and behavior. Journal of Experimental Social Psychology, 46, 792-798.

Lakens, D. (2013). Calculating and reporting effect sizes to facilitate cumulative science: A practical primer for t-tests and ANOVAs. Frontiers in Psychology, 4:863. doi:10.3389/fpsyg.2013.00863.

Lakens, D., \& Evers, E. (in press). Sailing from the seas of chaos into the corridor of stability: Practical recommendations to increase the informational value of studies. Perspectives on Psychological Science.

LeBoeuf, R. A., \& Estes, Z. (2004). “Fortunately, I'm no Einstein”: Comparison relevance as a determinant of behavioral assimilation and contrast. Social Cognition, 22, 607-636.

Loersch, C., \& Payne, B. K. (2011). The situated inference model: An integrative account of the effects of primes on perception, behavior, and motivation. Perspectives on Psychological Science, 6, 234-252.

Lowery, B. S., Eisenberger, N. I., Hardin, C. D., \& Sinclair, S. (2007). Long-term effects of subliminal priming on academic performance. Basic and Applied Social Psychology, 29, 151-157.

Macrae, C. N., Bodenhausen, G. V., Milne, A. B., Castelli, L., Schloerscheidt, A. M., \& Greco, S. (1998). On activating exemplars. Journal of Experimental Social Psychology, 34, 330-354.

McGuire, W. J. (1983). A contextualist theory of knowledge: Its implications for innovation and reform in psychological research. Advances in Experimental Social Psychology, $16,1-47$.

Mussweiler, T. (2006). Doing is for thinking! Stereotype activation by stereotypic movement. Psychological Science, 17, 17-21. 
Open Science Collaboration. (2012). An open, large-scale, collaborative effort to estimate the reproducibility of psychological science. Perspectives on Psychological Science, 7, 657-660. doi: 10.1177/1745691612462588

Nosek, B. A., \& Lakens, D. (in press). Registered reports: A method to increase the credibility of published results. Social Psychology.

Nussinson, R., Seibt, B., Häfner, M., \& Strack, F. (2010). Come a bit closer: Approach motor actions lead to feeling similar and behavioral assimilation. Social Cognition, 28, 4058.

Pashler, H., Coburn, N., \& Harris, C. R. (2012). Priming of social distance? Failure to replicate effects on social and food judgments. PLoS ONE, 7, e42510. doi:10.1371/journal .pone.0042510

Robinson, R. J., Keltner, D., Ward, A., \& Ross, L. (1995). Actual versus assumed differences in construal: "Naive realism" in intergroup perception and conflict. Journal of Personality and Social Psychology, 68, 404-417.

Sellke, T., Bayarri, M. J., \& Berger, J. O. (2001). Calibration of $p$ values for testing precise null hypotheses. The American Statistician, 55(1), 62-71.

Shanks, D. R., Newell, B. R., Lee, E. H., Balakrishnan, D., Ekelund, L., Cenac, Z., . . . Moore, C. (2013). Priming intelligent behavior: An elusive phenomenon. PLoS ONE, 8, e56515. doi:10.1371/journal.pone.0056515

Simmons, J. P., Nelson, L. D., \& Simonsohn, U. (2011). False positive psychology: Undisclosed flexibility in data collection and analysis allows presenting anything as significant. Psychological Science, 22, 1359-1366.

Simonsohn, U., Nelson L, Simmons, J. (2014). P-curve: A key to the file drawer. Journal of Experimental Psychology: General. 
P-Curving Professors and Elderly 18

Stroebe, W., \& Strack, F. (2014). Welcome back theory! Perspectives on Psychological Science, 9, 59-71. DOI: 10.1177/1745691613514450

Zwaan, R. A. (2014). Replications Should Be Performed With Power and Precision A Response to Rommers, Meyer, and Huettig (2013). Psychological Science, 25, 305307. 


\section{Footnotes}

${ }^{1}$ As with any meta-analytic procedure, it is important to justify the inclusion criteria for the performed analysis. I have included the studies discussed by Stroebe and Strack (2014) in the current analysis, because it provides an arguably complete overview of conceptually related replications in the published literature. Conceptual replications fall on a continuum, but any subjectivity in the selection of included studies can be attributed to authors arguing for rather than against the presence of priming effects. Finally, it would take an additional five conceptual replications similar to the best powered and most significant elderly priming study, and an additional eight conceptual replications similar to the best powered but barely significant professor priming study to change the conclusions about the evidential value of these research lines. 